\title{
ARTICLE
}

Molecular Diagnostics

\section{Quantification of HER1, HER2 and HER3 by time-resolved Förster resonance energy transfer in FFPE triple-negative breast cancer samples}

\author{
Alexandre Ho-Pun-Cheung ${ }^{1,2}$, Hervé Bazin ${ }^{3}$, Florence Boissière-Michot ${ }^{1}$, Caroline Mollevi ${ }^{1,2}$, Joëlle Simony-Lafontaine ${ }^{1}$, \\ Emeline Landas ${ }^{3}$, Jean-Pierre Bleuse ${ }^{1}$, Thierry Chardès ${ }^{2}$, Jean-François Prost ${ }^{4}$, André Pèlegrin ${ }^{2}$, William Jacot ${ }^{1,2}$, Gérard Mathis ${ }^{3}$ and \\ Evelyne Lopez-Crapez ${ }^{1,2}$
}

BACKGROUND: Triple-negative breast cancer (TNBC) has a worse prognosis compared with other breast cancer subtypes, and biomarkers to identify patients at high risk of recurrence are needed. Here, we investigated the expression of human epidermal receptor (HER) family members in TNBC and evaluated their potential as biomarkers of recurrence.

METHODS: We developed Time Resolved-Förster Resonance Energy Transfer (TR-FRET) assays to quantify HER1, HER2 and HER3 in formalin-fixed paraffin-embedded (FFPE) tumour tissues. After assessing the performance and precision of our assays, we quantified HER protein expression in 51 TNBC specimens, and investigated the association of their expression with relapse-free survival.

RESULTS: The assays were quantitative, accurate, and robust. In TNBC specimens, HER 1 levels ranged from $\approx 4000$ to more than 2 million receptors per cell, whereas HER2 levels varied from $\approx 1000$ to 60,000 receptors per cell. HER3 expression was very low (less than 5500 receptors per cell in all samples). Moderate HER2 expression was significantly associated with higher risk of recurrence $(\mathrm{HR}=3.93 ; P=0.003)$.

CONCLUSIONS: Our TR-FRET assays accurately quantify HER1, HER2 and HER3 in FFPE breast tumour specimens. Moderate HER2 expression may represent a novel prognostic marker in patients with TNBC.

British Journal of Cancer (2020) 122:397-404; https://doi.org/10.1038/s41416-019-0670-8

\section{BACKGROUND}

The term triple-negative breast cancers (TNBC) was first used in $2005^{1}$ to describe a subset of tumours characterised by absence or low levels of expression of oestrogen receptor (ER), progesterone receptor $(\mathrm{PgR})$, and human epidermal growth factor receptor 2 (HER2). The lack of ER, PgR and HER2 overexpression rules out the use of hormonal therapies or anti-HER2 agents in TNBC. For this reason, systemic treatment of TNBC was limited to chemotherapy till the recent introduction of poly-ADP ribose polymerase (PARP) inhibitors for BRCA-deficient tumours. ${ }^{2}$ Recurrence is more frequent and the 5-year survival rate is lower in patients with TNBC than other breast cancer subtypes (34\% versus $20 \%$, and $77 \%$ versus $93 \%$, respectively). ${ }^{3,4}$ Therefore, due to their poor prognosis and scarcity of targeted therapies, actionable molecular targets need to be identified.

Histologically, most TNBC share common characteristics, and $95 \%$ of them are classified as invasive ductal carcinomas. ${ }^{5}$ At the molecular level, Lehmann et al., ${ }^{6}$ and more recently, Burstein et al. ${ }^{7}$ have described molecular subtypes of TNBC with distinct outcomes and drug sensitivities. ${ }^{6-8}$ While additional studies are needed to determine whether the different TNBC subtypes can be targeted with specific therapies, these "omics" analyses demonstrated that TNBC should not be considered as a single clinical entity that can be uniformly treated.

Given TNBC molecular heterogeneity, targeting tumour-specific alterations could significantly improve the outcome of the $60-70 \%$ of patients with TNBC who do not fully respond to chemotherapy. ${ }^{5}$ As dysregulated expression of human epidermal receptor (HER) family members is frequent in breast cancer, and given their crucial role in proliferation, ${ }^{9}$ these receptors have been extensively investigated as targets for anticancer therapy, particularly HER1, HER2 and HER3. HER1 overexpression is frequent in TNBC and is associated with poor clinical outcome. ${ }^{10}$ Preclinical studies demonstrated the sensitivity of TNBC cell lines to HER1 inhibitors, providing the rationale to test the efficacy of HER1-targeting agents in patients with TNBC. ${ }^{11}$ However, clinical studies remain inconclusive, possibly due to the lack of patient selection, because anti-HER1 targeted therapies were not restricted to patients with HER1-overexpressing tumours or patients without KRAS/NRAS mutations. $^{12}$ Some recent studies suggested that patients with TNBC, which is, by definition, characterised by absence of HER2 overexpression, could benefit from anti-HER2 therapies, such as trastuzumab. ${ }^{13,14}$ This issue was specifically addressed by the National Surgical Adjuvant Breast and Bowel Project B-47 (NSABP

\footnotetext{
${ }^{1} \mathrm{ICM}$, Institut régional du Cancer de Montpellier, Montpellier, France; ${ }^{2}$ Institut de Recherche en Cancérologie de Montpellier (IRCM), INSERM, Université de Montpellier, Institut régional du Cancer de Montpellier (ICM), Montpellier, France; ${ }^{3}$ CisBio SA, Codolet, France and ${ }^{4}$ GamaMabs Pharma SA, Centre Pierre Potier, Toulouse, France Correspondence: Evelyne Lopez-Crapez (evelyne.crapez@icm.unicancer.fr)

These authors contributed equally: Alexandre Ho-Pun-Cheung, Hervé Bazin
}

Received: 20 May 2019 Revised: 5 November 2019 Accepted: 13 November 2019

Published online: 3 December 2019 
B-47) trial that was designed to evaluate trastuzumab effect in 3270 women with breast cancers with low HER2 levels (IHC $1+$ or $2+$ and/or negative by FISH). ${ }^{15}$ This randomised trial did not find any improvement in invasive disease-free survival or overall survival in patients treated with trastuzumab and standard chemotherapy versus chemotherapy alone. ${ }^{16}$ Nevertheless, a recent study reported that in patients with TNBC, moderate HER2 expression (IHC score of $2+$ ) correlates with relapse, ${ }^{17}$ suggesting that the clinical significance of different degrees of HER2 expression should be thoroughly explored in TNBC. Finally, the therapeutic potential of anti-HER3 agents for cancer treatment has been less investigated because HER3 kinase domain is defective. However, HER3 expression could be a prognostic marker in TNBC. ${ }^{18}$

IHC has become the gold standard method to assess protein expression in formalin-fixed, paraffin-embedded (FFPE) clinical samples. However, IHC typically relies on chromogenic detection that has a narrow linear dynamic range, thus limiting its ability to generate accurate quantitative results. ${ }^{19}$ Moreover, IHC scoring system is subjective and this can lead to reproducibility issues. ${ }^{20,21}$ Therefore, to improve the accuracy and reproducibility of HER family member quantification in tumours, we developed a timeresolved Förster resonance energy transfer (TR-FRET) method that allows the quantitative and objective measurements of protein expression levels. This method relies on the energy transfer between two fluorophores, a donor and an acceptor. ${ }^{22}$ When the donor (in this case, a lanthanide complex characterised by a long fluorescence lifetime) is excited by an energy source, it transfers its excitation energy to the acceptor only if the two fluorophores are in close proximity. The acceptor will then emit a specific long-lived fluorescence. In our assays, the analytes are detected using two antibodies that bind to two distinct epitopes within the analyte. One antibody is coupled to a donor fluorophore, while the second antibody is coupled to an acceptor fluorophore. When these antibodies bind to specific epitopes on the target protein, the distance between the donor and the acceptor is small enough to allow the energy transfer. The intensity of the acceptor fluorescence signal is proportional to the receptor number in the sample or standard, thereby allowing quantitative measurements. We previously described such assays for the quantification of HER1 and HER2 in tumour cryosections. ${ }^{23,24}$ As the use of these TR-FRET assays is limited by the need of fresh or freshly frozen tumour tissues, we now developed new TR-FRET assays to quantify HER1, HER2 and HER3 in FFPE tumour samples. In this study, we evaluated HER quantification by TR-FRET assays and assessed the prognostic role of HER1, HER2 and HER3 expression in TNBC.

\section{METHODS}

Patients and tissue samples

Tumour samples were selected from the Montpellier Cancer Institute (ICM) biological resource centre. Pathological data including hormone-receptor status, HER2 status, histological type, grade and PTNM were recorded. Clinical data (e.g. age, type of treatment, occurrence and type of relapse) were obtained by review of the medical files. The selected population included patients with non-metastatic TNBC who underwent mastectomy or breast-conservative surgery with negative margins, without previous history of cancer. In total, 51 patients with TNBC treated between 2004 and 2008 were included in this retrospective study. These patients did not receive neoadjuvant treatment and were treated by postoperative radiotherapy or by radiotherapy with standard chemotherapy (anthracyclines and/or taxanes). All tumours were considered as ER- and PgR-negative and HER2unamplified. ER and PgR status were assessed by IHC, using the mouse monoclonal antibodies 6F11 (against ER, 1:100 dilution, Leica, Wetzlar, Germany) and PgR636 (against PgR, 1:400 dilution,
Dako, Glostrup, Denmark). ER and PgR negativity were defined as $<10 \%$ of ER and PgR immunoreactivity, irrespective of the staining intensity. Of note, among the 51 ER-negative tumours, 5 included $1-10 \%$ of ER-stained tumour cells. HER2 status was determined by IHC using the anti-cErbB2 polyclonal antiserum (\#A0485, 1:800 dilution, Dako). HER2 expression was scored according to the recommendations at the time of diagnosis. ${ }^{25}$ HER2 negativity was defined as a score of $0,1+$, or $2+$, with normal gene copy number by fluorescent/chromogenic in situ hybridisation in tumours with a $2+$ score.

\section{Tissue lysate preparation}

For each FFPE tumour sample, a $3 \mu \mathrm{m}$-thick section was examined histologically after haematoxylin and eosin staining to assess the percentage of tumour cells. Adjacent $5 \mu \mathrm{m}$-thick sections were then macro-dissected to enrich in tumour cells $(>50 \%)$ and used for lysate preparation. The number of sections was adjusted to the tumour area (ideally three sections for a tumour with an area of $100 \mathrm{~mm}^{2}$ after macro-dissection). Sections were placed in a $1.5 \mathrm{ml}$ microcentrifuge tube containing $1 \mathrm{ml}$ of xylene substitute (\#A5597, Sigma, lle d'Abau, France). Paraffin was dissolved by incubation at room temperature (RT) for $5 \mathrm{~min}$, and then removed by centrifugation. Pellets were washed to remove residual contaminants (absolute ethanol twice, then 95\% ethanol), collected by centrifugation (12,000 RCF) and resuspended in $1 \mathrm{X}$ Tris/EDTA pH 9 (Target Retrieval Solution \#S2367, Dako). Samples were then heated at $95^{\circ} \mathrm{C}$ in a Thermomixer (Eppendorf, Hamburg, Germany) for 45 min, centrifuged to remove the Tris/ EDTA buffer, and resuspended in $250 \mu$ l of Lysis Buffer (LB4 \#64KL4FDF, Cisbio, Codolet, France). Finally, ice-cold samples were lysed by sonication ( $80 \mathrm{~W}$ for $15 \mathrm{~s}$ ) using a Vibra-Cell ${ }^{\mathrm{TM}}$ sonicator (Sonics \& Materials, Newtown, CT, USA). Lysates were clarified by centrifugation (10,000 RCF) at $4{ }^{\circ} \mathrm{C}$ for $5 \mathrm{~min}$ and then transferred to the wells of a microtiter plate.

\section{TR-FRET assays}

The TR-FRET assays were carried out in white 384-well small volume high-base microtiter plates (Ref. 784075 Greiner Bio-One, Courtaboeuf, France) in duplicate using $\mathrm{Eu}^{3+}$ cryptate trisbipyridine (TBP, \#62EUSPEA, Cisbio) as donor, and d2 as acceptor, conjugated to the specific antibodies. Antibodies were labelled as previously described. ${ }^{24}$ For HER1 quantification, the TR-FRET assay was performed using two monoclonal antibodies from the Total EGFR Cellular Assay Kit (\#64NG1PEG, Cisbio). HER2 expression was quantified using Ab-15 (LabVision, Fremont, CA, USA) labelled with TBP, and Ab-8 (LabVision) labelled with d2. HER3 was quantified using the ErbB3 monoclonal antibody 2F12 (Thermo Scientific, Rockford, IL, USA) labelled with TBP, and the ErbB3 monoclonal antibody 2B5 (Thermo Scientific) labelled with $\mathrm{d} 2$. The final assay volume was $20 \mu \mathrm{l}$ and comprised $16 \mu \mathrm{l}$ of lysate and $4 \mu \mathrm{l}$ of a solution containing the donor/acceptor antibodies diluted in HTRF cellular kinase detection buffer (Cisbio). Plates were incubated at RT for $20 \mathrm{~h}$, and read using a Pherastar FS fluorometer (BMG LABTECH, Champigny-sur-Marne, France) with a classical HTRF protocol (excitation at $337 \mathrm{~nm}$, donor and acceptor emission measured, respectively, at $620 \mathrm{~nm}$ and $665 \mathrm{~nm}, 60 \mu$ s delay, $400 \mu \mathrm{s}$ integration). The TR-FRET signals (deltaF) were calculated as previously described. ${ }^{26}$

For each assay, a standard curve was prepared using human recombinant proteins to convert the TR-FRET signals into receptor number per $\mu$ l of lysate. Recombinant HER1, HER2 and HER3 were purchased from Origene technologies (Catalog No.: TP710011; TP710032 and TP710089, respectively).

For normalisation to DNA concentration, $2 \mu \mathrm{l}$ of the sample solution used for the TR-FRET assays was transferred into a new microplate well, and $18 \mu \mathrm{l}$ of a solution containing 1 X SYBR Safe DNA Gel Stain (Invitrogen, Carlsbad, CA, USA), 50 mM HEPES buffer and $0.1 \%$ BSA was added. After incubation at RT for $2 \mathrm{~h}$, SYBR 
Green fluorescence intensity was measured by reading the fluorescence at $520 \mathrm{~nm}$ in Fluorescence Polarization mode upon $485 \mathrm{~nm}$ excitation using a Pherastar FS fluorimeter. Sample DNA concentrations were interpolated from a standard curve generated using serial dilutions in LB4 of a DNA standard of known concentration $(50 \mu \mathrm{g} / \mathrm{ml})$ prepared from human placental DNA (\# D-7011, Sigma). By assuming 6.6 pg DNA per diploid cell, $1 \mathrm{ng}$ of DNA was considered to correspond to 151 cells. This allowed converting the number of receptors per ng of DNA into a number of receptors per cell.

Immunohistochemistry assays

IHC analysis was performed on $3 \mu \mathrm{m}$-thick FFPE tumour tissue sections. The HER1 and HER3 IHC assays were carried out on adjacent sections that are the mirror image of the ones used for the TR-FRET assays. For HER1 detection, slides were deparaffinised in xylene, hydrated in serial dilutions of alcohol, and then immersed in proteinase K solution (Dako) at RT for $10 \mathrm{~min}$. For HER3 detection, section deparaffinisation, rehydration and antigen retrieval were performed simultaneously in an automated PT Link module (Dako) using EnVision FLEX Target Retrieval Solution, High $\mathrm{pH}$ (Dako), at $95^{\circ} \mathrm{C}$ for $15 \mathrm{~min}$. Endogenous peroxidase activity was blocked by incubation with the EnVision Flex Peroxidase Block (Dako) at RT for 5 min. Sections were then incubated with anti-HER1 (mouse monoclonal, clone 31G7, 1:50 dilution, Invitrogen) or anti-HER3 (mouse monoclonal, clone DAK-H3-IC, 1:50 dilution, Dako) antibodies, for 20 and $30 \mathrm{~min}$, respectively. For HER3 detection, EnVision FLEX + Mouse LINKER (Dako) was used to amplify the primary antibody signal. After two rinses in EnVision FLEX wash buffer (Dako), sections were incubated with a horseradish peroxidase-labelled polymer coupled to secondary antibodies (Envision FLEX HRP, Dako) at RT for 20 min, followed by incubation with EnVision FLEX Substrate Working Solution containing 3,3'-Diaminobenzidine as chromogen (Dako) at RT for 10 min. Sections were counterstained with EnVision Flex Hematoxylin (Dako), rinsed with tap water for $5 \mathrm{~min}$, dehydrated, and mounted with a coverslip. A negative control (a sample incubated with non-specific mouse lgG instead of the primary mouse monoclonal antibodies) was included in each IHC batch. Sections were read independently by two trained observers (BoissièreMichot $\mathrm{F}$ and Simony-Lafontaine J) blinded to the patients' clinicopathological characteristics. HER1 and HER3 expression levels were scored using the $\mathrm{H}$-Score method ${ }^{27}$ in which the membrane staining intensity (no staining $=0$, weak staining $=1$, moderate staining $=2$ and intense staining $=3$ ) is multiplied by the percentage of stained tumour cells (from 0 to 100\%) to give a score ranging from 0 to $300 . \mathrm{H}$-Scores were averaged between observers, except for conflicting results that were examined conjointly to reach consensus. HER2 expression levels were extracted from the patient medical files, and therefore, they were from IHC assays carried out using sections that were not the mirror image of the ones used for the TR-FRET assays.

Statistical analysis

Continuous variables were described as medians and range, and categorical variables as frequencies and percentages. A nonparametric test for trend was performed to test the TR-FRET expression increase across the three ordered groups of expression determined by IHC (weak/negative, moderate, and high for HER1 and HER3; 0, 1+, and 2+ for HER2). Relapse-free survival (RFS) was defined as the time from the date of surgery to the date of last contact or recurrence (local, regional, or distant), and estimated using the Kaplan-Meier method. Univariate and multivariate analyses were performed using a Cox proportional hazard model. Hazard ratios (HR) were given with their $95 \%$ confidence interval $(95 \% \mathrm{Cl})$. All statistical analyses were performed with the STATA 13.0 software (StatCorp, College Station, TX).

\section{RESULTS}

Patients and clinicopathological characteristics

The study cohort included 51 women with TNBC. Using 20 November 2014 as cut-off date, the median follow-up was 6.5 years $(95 \% \mathrm{Cl}$ [5.3-7.8]). Among these patients, 19 (37.2\%) had a recurrence that was loco-regional in nine and distant in ten patients. Most of these recurrences (84\%) occurred during the first 42 months of follow-up, which is consistent with the previously reported relapse risk temporal distribution. ${ }^{3}$ The patients' clinicopathological characteristics are detailed in Table 1.

\section{TR-FRET assay performance}

The standard curves for TR-FRET-based quantification of HER expression showed a linear relationship between TR-FRET signal and HER1, HER2 and HER3 concentration for concentration ranges of $0.98-250 \mathrm{ng} / \mathrm{ml} \quad\left(R^{2}=0.995\right), 0.24-62.5 \mathrm{ng} / \mu \mathrm{l} \quad\left(R^{2}=0.997\right)$ and $0.24-62.5 \mathrm{ng} / \mathrm{ml}\left(R^{2}=0.988\right)$, respectively, and tended to saturate at higher levels (Fig. 1). The limit of detection (LOD) was in the range of $0.19-0.60 \mathrm{ng} / \mathrm{ml}$, depending on the assay. The lower limit of quantification (LLOQ) values were $0.98 \mathrm{ng} / \mathrm{ml}$ (HER1), $0.48 \mathrm{ng} / \mathrm{ml}$ (HER2), and $0.48 \mathrm{ng} / \mathrm{ml}$ (HER3). For all assays, the upper limit of quantification (ULOQ) was equal to the concentration of the highest calibration standard within the linear dynamic range of concentrations (HER1: $250 \mathrm{ng} / \mathrm{ml}$; HER2: $62.5 \mathrm{ng} / \mathrm{ml}$; HER3: $62.5 \mathrm{ng} / \mathrm{ml}$ ).

As the TR-FRET assays rely on antigen-antibody interactions, the specificity of each antibody pair needed to be validated. For that purpose, the ability of the antibody pairs to specifically detect HER1, HER2 or HER3 was tested by using as negative controls the other HER family members. These negative controls $(1000 \mathrm{ng} / \mathrm{ml}$ of recombinant proteins) did not generate any TR-FRET signal above the LOD (data not shown).

Precision of the TR-FRET assays

For each of the 51 TNBC samples, two independent technical replicates were prepared using consecutive serial sections from the same FFPE tumour block. Each lysate was independently used for HER1, HER2 and HER3 quantification by TR-FRET. Technical replicate data were normalised and the numbers of receptors per cell were then plotted to assess the precision of each assay. The values of duplicate samples were strongly correlated (HER1: $R^{2}=$ 0.986; HER2: $R^{2}=0.993$; HER3: $R^{2}=0.877$ ) (Supplementary Fig. 1).

Table 1. Patients' characteristics.

\begin{tabular}{ll}
\hline Characteristics & Number of patients (\%) \\
\hline Age, years & \\
Median & 62 \\
Range & $30-89$ \\
TNBC histological type & \\
Ductal & $46(90.2)$ \\
Other & $5(9.8)$ \\
Histologic grade (SBR) & \\
I & $1(2.0)$ \\
II & $8(15.7)$ \\
III & $42(82.3)$ \\
pT stage & \\
T1 & $14(27.4)$ \\
T2 & $33(64.7)$ \\
T3 & $3(5.9)$ \\
T4 & $1(2.0)$ \\
pN stage & \\
N- & $25(49.0)$ \\
N+ & $26(51.0)$ \\
\hline
\end{tabular}



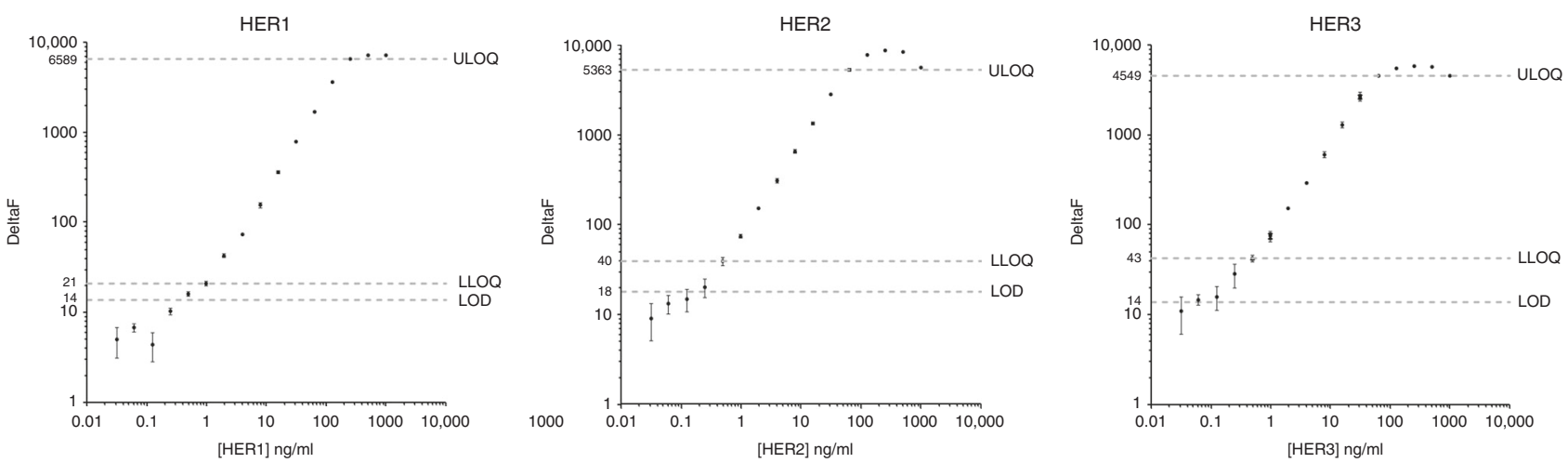

Fig. 1 Calibration curve plots showing the limit of detection (LOD), lower limit of quantification (LLOQ), linear dynamic range, and upper limit of quantitation (ULOQ) of the HER1, HER2 and HER3 TR-FRET assays. For each assay, the LOD was calculated as the average signal of 20 replicate-negative samples plus three standard deviations. The lower limit of quantification (LLOQ) and the upper limit of quantification (ULOQ) were the lowest and highest standard point that can be measured with $<15 \%$ coefficient of variance (CV).

\begin{tabular}{|c|c|}
\hline & Number of patients (\%) \\
\hline \multicolumn{2}{|l|}{ HER1 Expression } \\
\hline Weak (H-Score < 10) & $22(43.1 \%)$ \\
\hline Moderate $(10 \leq \mathrm{H}$-Score $<150)$ & $22(43.1 \%)$ \\
\hline High (H-Score $\geq 150$ ) & $7(13.8 \%)$ \\
\hline \multicolumn{2}{|l|}{ HER2 Expression } \\
\hline 0 & $40(78.4 \%)$ \\
\hline $1+$ & $9(17.7 \%)$ \\
\hline $2+$ & $2(3.9 \%)$ \\
\hline $3+$ & $0(0 \%)$ \\
\hline \multicolumn{2}{|l|}{ HER3 Expression } \\
\hline Weak (H-Score < 10) & $42(82.4 \%)$ \\
\hline Moderate $(10 \leq \mathrm{H}$-Score < 150$)$ & $9(17.6 \%)$ \\
\hline High (H-Score $\geq 150$ ) & $0(0 \%)$ \\
\hline
\end{tabular}

The median coefficient of variation values between technical replicates were $5.3 \%$ (HER1), 5.2\% (HER2), and $8.2 \%$ (HER3). Taken together, these results demonstrate that the TR-FRET assays allow the reliable and precise quantification of HER1, HER2 and HER3 in FFPE tumour samples.

HER1, HER2 and HER3 expression in TNBC

The IHC results are presented in Table 2 and in Supplementary Table 1. Briefly, among the 51 TNBC specimens, only seven (13.8\%) displayed high HER1 expression. In most tumours, HER2 could not be detected (score $=0)$, and only 11 TNBC samples $(21.5 \%)$ showed weak or moderate HER2 expression (score $=1+$ or $2+$ ). Moderate HER3 expression was found in nine tumours (17.6\%), and none showed high HER3 expression. Representative examples of HER3 IHC staining are shown in Supplementary Fig. 2. Although only membrane staining was taken into account in the H-Score, HER3 membrane expression was significantly associated with cytoplasmic expression $(P<0.001)$. Indeed, 22 of the 30 HER3 membrane-positive tumours displayed concomitant cytoplasmic staining, whereas only 2 of the 21 HER3 membrane-negative TNBC samples showed HER3 cytoplasmic expression. HER3 nuclear expression was detected to various extent in 53\% of TNBC specimens (27 of 51), without association with membrane or cytoplasmic HER3 expression (data not shown).

The HER1, HER2 and HER3 expression levels measured by TRFRET are shown in Fig. 2, and data are listed in Supplementary

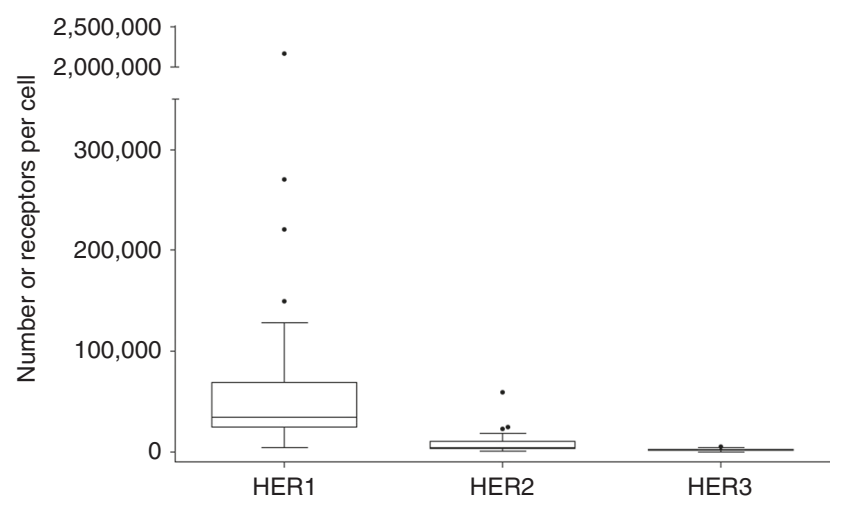

Fig. 2 Quantitative measurement of HER1, HER2 and HER3 expression by TR-FRET in 51 TNBC samples.

Table 1. The median number of receptors per cell were 34,540 (range 4452-2,159,308) for HER1, 4463 (range 945-59,302) for HER2, and 1532 (323-5348) for HER3. Low endogenous HER1, HER2 and HER3 levels (below 10,000 receptors per cell) were observed in 2 (3.9\%), 37 (72.6\%) and 51 (100.0\%) tumours, respectively. Moderate expression (between 10,000 and 100,000 receptors per cell) of HER1 and HER2 was observed in 43 (84.3\%) and $14(27.4 \%)$ tumours, respectively. High HER1 expression (above 100,000 receptors per cell) was found in six $(11.8 \%)$ tumours. Not surprisingly, in most tumours, HER1 expression levels were more elevated than those of HER2 and HER3 (96 and 100\% of tumours, respectively).

Comparison of the TR-FRET and IHC results (Supplementary Fig. 3) showed a significant trend of increasing HER1 $(P<0.001)$ and HER2 $(P=0.007)$ receptor number by TR-FRET across the ordered IHC expression groups. For HER3, no significant trend was found between IHC and TR-FRET expression levels, because of its very low expression.

Correlation with relapse-free survival

The estimated 36-month RFS rate was 76\% (95\% Cl 62-86\%). Univariate analysis showed that histological type, histologic grade and pathologic stage (pT) were not correlated with the RFS rate. Patients with node-positive $(\mathrm{pN}+)$ TNBC were more likely to relapse, although this trend did not reach significance $(P=0.056)$. Analysis of whether the HER1, HER2 or HER3 expression levels could be potential prognostic factors of relapse showed that moderate HER2 expression, assessed by IHC $(P=0.043)$ or TR-FRET $(P=0.003)$, was associated with significantly reduced RFS (Table 3 and Fig. 3 ). When 
Table 3. Univariate and multivariate analyses of prognostic factors related to relapse-free survival.

\begin{tabular}{|c|c|c|c|c|c|c|c|}
\hline Prognostic marker & Events/patients (n) & \multicolumn{3}{|c|}{ Univariate analysis } & \multicolumn{3}{|c|}{ Multivariate analysis } \\
\hline $\mathrm{pN}$ & & & & 0.056 & & & \\
\hline $\mathrm{pN}-$ & $6 / 25$ & 1 & & & & & \\
\hline $\mathrm{pN}+$ & $13 / 26$ & 2.47 & $0.94-6.51$ & & & & \\
\hline HER2 expression (TR-FRET) & & & & 0.003 & & & 0.003 \\
\hline HER2 expression (IHC) & & & & 0.043 & & & \\
\hline 0 & $12 / 40$ & 1 & & & & & \\
\hline $1+/ 2+$ & $7 / 11$ & 2.78 & $1.09-7.10$ & & & & \\
\hline
\end{tabular}

HER2 expression levels measured by TR-FRET were dichotomised in the low and moderate expression groups according to the cut-off value derived from the ROC curve for predicting disease recurrence or metastases. The optimal cut-off value was 8,500 receptors per cell (sensitivity $=52.63 \%$, specificity $=84.38 \%$ ). aLikelihood-ratio test.
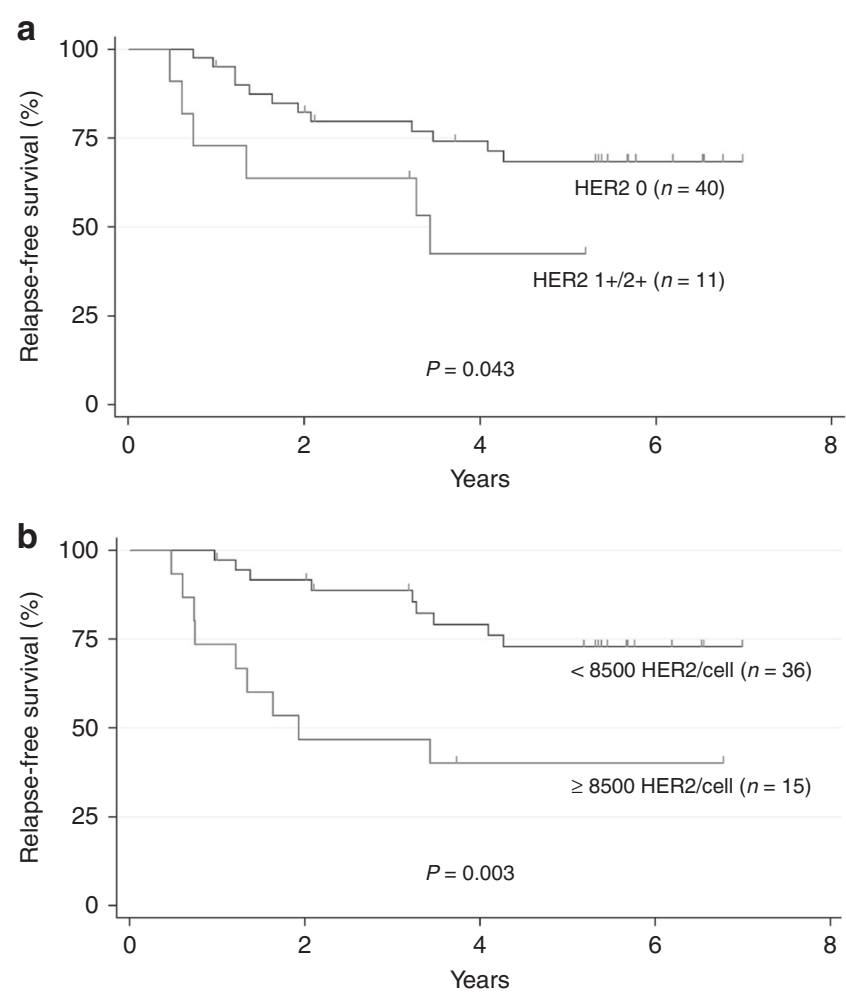

Fig. 3 Kaplan-Meier curves for relapse-free survival of 51 patients with TNBC stratified according to HER2 expression by IHC (a) and by TR-FRET (b).

variables with $p<0.1$ were entered in multivariate analyses, only HER2 TR-FRET expression was an independent risk factor for tumour recurrence (Table 3 ).

\section{DISCUSSION}

Recently, we described a TR-FRET-based assay for the precise and objective quantitative analysis of HER family member expression in tumour cryosections. ${ }^{23}$ Here, we successfully applied this TRFRET technology for the quantification of HER1, HER2 and HER3 in FFPE tumour samples.

The use of standard curves generated with recombinant HER1, HER2 and HER3 proteins allowed us to convert TR-FRET signals into number of receptors per $\mu \mathrm{l}$ of lysate. However, to accurately compare protein expression levels between patient samples using a quantitative method, measurements must be normalised relatively to the amount of starting material. Ideally, cell number should be assessed for each sample to derive the amount of proteins per cell. While this approach is feasible for cultured cells and blood samples, it is not applicable to tissue lysates. As DNA concentration is a robust surrogate of cell number in a wide range of cell types, including cancer cell lines, ${ }^{28}$ we developed a SYBR green-based quantification assay that measures the total DNA concentration in the same reaction mix used for the TR-FRET assay. Although most cancer cells are not diploid, we assumed that $1 \mathrm{ng}$ of DNA was equivalent to 151 cells. ${ }^{29}$ For each assay, we then derived the number of receptors per cell from the number of receptors per ng of DNA. It is important to note that several factors could influence the determination of the receptor number per cell. First, chromosomal abnormalities are a hallmark of cancer cells, which means that tumour cells have variable DNA content. In addition, for tissue lysate preparation, samples undergo physical disruption in denaturing conditions that can damage HER protein epitopes. This implies that the measured receptor number is likely to represent an underestimation of the true receptor number. Finally, the extraction and solubilisation efficiency might be different between proteins and DNA. For these reasons, the calculated number of receptors per cell may differ from the true number of receptors per cell. Nevertheless, the TR-FRET signal conversion into number of receptors per cell is very convenient because it makes it easier to visualise the receptor expression level (low, moderate, or high) in a tumour. Moreover, the calculated number of receptors per cell was consistent from experiment to experiment, as indicated by the low coefficient of variation $(\mathrm{CV}<10 \%)$ between technical replicates prepared from adjacent, mirror-image FFPE sections. This standardised relative quantification allows comparing the expression levels of different proteins, which is not possible with a semi-quantitative method, such as IHC.

We determined the performance of the new HER1, HER2 and HER3 TR-FRET assays for FFPE tumour samples by determining their LOD (from 0.19 to $0.60 \mathrm{ng} / \mathrm{ml}$ ) and dynamic range (250-fold linear dynamic range). By comparison, commercially available ELISA kits, such as the Invitrogen human EGFR, ErbB2 and ErbB3 ELISA kits (Catalogue No.: KHR9061; EHERBB2 and EHERBB3, respectively), are more sensitive $(<0.1 \mathrm{ng} / \mathrm{ml})$, but with smaller dynamic range (less than 100 -fold), and require much more biological material (sample volume is usually $100 \mu \mathrm{l}$ vs $16 \mu \mathrm{l}$ for our TR-FRET assays). Moreover, these ELISA kits are intended for serum 
402

and cell culture supernatants, and may not be suitable for HER1, HER2 or HER3 quantification in FFPE lysates.

When used to quantify HER1, HER2 and HER3 in 51 FFPE TNBC samples, our TR-FRET approach demonstrated a high reproducibility level (median CV of technical replicates $\leq 8 \%$ ). This compares quite favourably with $\mathrm{CV}$ of $\approx 15 \%$ reported for other quantitative methods to measure protein expression in FFPE samples, such as the mTRAQ $^{30}$ and VeraTag ${ }^{\text {TM }}$ proximity-based assays. ${ }^{31}$ We think that due to their precision, sensitivity and objectivity, our TR-FRET assays are an appropriate method for the quantification of HER family members.

The HER family plays a major role in the regulation of cell proliferation, differentiation, and survival (9), and aberrant HER signalling is frequent in breast cancer. To clarify the role of HER1, HER2 and HER3 in TNBC, we assessed the expression of these receptors in our 51 TNBC using IHC and compared the results with those obtained with the TR-FRET assays.

HER1 IHC overexpression has been reported in 13-76\% of TNBC $^{32}$ and is associated with worse disease-free survival. ${ }^{10}$ HER1 overexpression frequency depends strongly on the scoring methods and the antibodies used. ${ }^{33}$ In our study, we detected high HER1 expression only in $13.8 \%$ of TNBC samples by IHC with the anti-HER1 antibody clone $31 \mathrm{G} 7$ (Dako), which is widely used to assess HER1 expression in colorectal cancer, and the H-Score scoring method. We obtained similar results with the TR-FRET assay $(11.8 \%$ of tumours with $>100,000$ HER 1 receptors per cell). However, HER1 number per cell was higher than 10,000 HER1 in most TNBC samples, a value above the level that is usually considered as endogenous/physiological. ${ }^{34,35}$ These results nuance rather than contradict the belief that HER1 overexpression is common in TNBC.

Currently, HER2 status is generally determined by IHC. In our series of 51 TNBC, comparison of the TR-FRET and IHC results showed a significant trend of increasing HER2 receptor number by TR-FRET across the HER2 IHC ordered groups, from 0 to $2+$. However, our HER2 TR-FRET assay could detect as few as 900 receptors per cell, whereas conventional IHC is typically 20 times less sensitive. ${ }^{34}$ HER2 expression values varied considerably among TNBC samples, with tumours displaying up to 60,000 receptors per cell. Therefore, in some TNBC, HER2 expression level is not negligible. Of note, the Ab-8 and Ab-15 antibodies used for HER2 TR-FRET quantification recognise epitopes localised in HER2 intracellular domain. This means that our HER2 TR-FRET assay also detects p95HER2, a truncated form of HER2 that lacks the extracellular domain.

HER3 overexpression has been associated with poor outcome in many cancer types. ${ }^{36,37}$ In our 51 TNBC samples, $82.4 \%$ displayed negative or weak HER3 expression by IHC, and all tested samples had less than 5500 receptors per cell by TR-FRET analysis. Our results indicate that most TNBC have very low HER3 expression.

Finally, analysis of HER1, HER2 and HER3 expression patterns relative to the patient outcome showed that HER2 level is a potential biomarker of relapse. Indeed, moderate HER2 expression by IHC (score of $1+/ 2+$ ) and TR-FRET ( $\geq 8500$ HER2 per cell) was significantly associated with reduced RFS in our cohort of 51 TNBC. These results are in accordance with previous findings showing that moderate HER2 expression (IHC score of $2+$ ) correlates with relapse in a small series of 47 patients with TNBC. ${ }^{17}$ This suggests that HER2 could be an actionable target in TNBC with moderate HER2 expression levels. Two controversial studies published in $2008^{13}$ and $2010^{14}$ showed that some patients with HER2-negative breast tumours could benefit from HER2-targeting agents. Ithimakin et al. suggested that this unexpected finding might be explained by the cancer stem cell (CSC) hypothesis ${ }^{38}$ according to which CSCs are implicated in treatment resistance ${ }^{39,40}$ and tumour recurrence. ${ }^{41}$ Specifically, Ithimakin et al. demonstrated that, in some tumours with moderate HER2 levels, HER2 is selectively expressed in the CSC population. Therefore, trastuzumab and other HER2-targeting agents should be effective by targeting HER2-expressing CSCs. Indeed, trastuzumab can reduce the CSC populations of cell lines with moderate HER2 levels by specifically targeting HER2, and when administered early (adjuvant setting), it blocks the growth of tumour xenografts of these cell lines. Conversely, trastuzumab has no effect on cell lines and xenografts with low HER2 expression. ${ }^{38}$ However, the recent results of the NSABP B-47 trial undermine this theory by showing that adjuvant trastuzumab in patients with HER2 $1+$ or $2+$ breast cancers by IHC does not improve disease- or relapse-free survival in this population. ${ }^{16}$ One possible explanation is that moderate HER2 expression is challenging to detect by IHC because of its semiquantitative nature and its narrow dynamic range. In our study, multivariate analysis demonstrated that HER2 protein expression determined by TR-FRET is a stronger predictor of relapse than the HER2 IHC score. However, these results have some limitations due to the small patient population and the retrospective nature of our study. Prospective studies with larger cohorts of patients are required to confirm the relevance of our results.

Currently, IHC is the gold standard method to assess protein expression in patient tissue samples; however, its reproducibility is still an issue, although many improvements have been made in the past several years. ${ }^{21}$ Alternative methods exist for the objective quantification of HER proteins in FFPE samples. First, IHC can be enhanced by using fluorescence microscopy and advanced image analysis algorithms. This approach allows the continuous and quantitative measure of protein expression, but the inherent autofluorescence of FFPE sections may be a source of trouble. ${ }^{42}$ Recently, Targeted Mass Spectrometry has been used to assess HER2 expression in FFPE tissue samples. ${ }^{43}$ This approach is more quantitative than IHC and does not depend on the availability of specific antibodies. However, the detection of low and moderate levels of proteins remains challenging. Alternatively, Monogram Biosciences proposes the VeraTag ${ }^{\text {TM }}$ proximitybased assays ${ }^{44,45}$ to quantify HER1, HER2 and HER3 in FFPE samples. The VeraTag ${ }^{\mathrm{TM}}$ technology uses a dual antibody format, whereby a fluorescent tag on one anti-HER antibody is released when in close proximity to a second specific antibody conjugated with molecular scissors that are activated upon illumination. The VeraTag $^{\mathrm{TM}}$ assays are very sensitive and can detect down to 2500 receptors per cell. ${ }^{46}$ However, access to this technology for research purposes is restricted because samples must be shipped to the Monogram CAP/CLIA certified laboratory in California for the analysis. We believe that our TR-FRET approach is a suitable alternative to these methods, because it overcomes many of their limitations. By using long-lived fluorophores, a delay can be introduced between the excitation pulse and the signal measurement window, thus allowing the elimination of short-lived background autofluorescence from FFPE material. Our assays are quantitative, with high sensitivity, and could be easily performed in any laboratory equipped with a TR-FRET instrument, without much training required. Moreover, the TR-FRET technology could be extended to the quantification of other proteins of interest, such as p95HER2, the relevance of which as a prognostic marker has recently been reported in patients with HER2-positive breast cancer treated with trastuzumab. ${ }^{47,48}$

In summary, the present study demonstrates that our TR-FRET assays can reproducibly quantify the expression of HER family members in FFPE samples with high sensitivity. Moreover, it shows that quantification of HER2 expression by TR-FRET may be useful to predict tumour recurrence in TNBC, although additional studies in a larger population are required to confirm our findings.

\section{ACKNOWLEDGEMENTS}

We thank Magalie Pedot for her excellent technical assistance. We are grateful to Blandine Massemin for her help in collecting and preparing clinical samples. We also thank Sonia Boulabas for collecting the clinical data. 


\section{AUTHOR CONTRIBUTIONS}

E.C., T.C., J.F., A.P. and G.M. were responsible for the conception and design of the study. J.B. and W.J. enrolled the patients and contributed to the patient data collection. A.H., H.B., F.B., E.L. and J.S. performed the experiments. A.H., C.M., E.C. and F.B. contributed to data analysis and interpretation. A.H. drafted the manuscript and prepared the figures. All authors discussed the results and implications, and critically revised and approved the final manuscript version.

\section{ADDITIONAL INFORMATION}

Ethical approval and consent to participate The present study was performed in accordance with the Declaration of Helsinki guidelines, and was approved by the ICM Institutional Review Board (ID number ICM-CRB-2012/04). Samples were provided by ICM biological resource centre (Biobank number BB-0033-00059), following the ethics and legal national French dispositions for the patients' information and consent. Considering the retrospective, non-interventional nature of this study, no consent was deemed necessary by the review board.

Consent for publication Our manuscript does not contain any individual person's data in any form.

Data availability The datasets generated and/or analysed during the current study are available from the corresponding author on reasonable request.

Competing interests The authors declare no competing interests.

Funding information This work was supported by the grant AAP13 "Fonds Unique Interministériel" FUI UmAbHER3 F120402M.

Supplementary information is available for this paper at https://doi.org/10.1038/ s41416-019-0670-8

Note This work is published under the standard license to publish agreement. After 12 months the work will become freely available and the license terms will switch to a Creative Commons Attribution 4.0 International (CC BY 4.0).

Publisher's note Springer Nature remains neutral with regard to jurisdictional claims in published maps and institutional affiliations.

\section{REFERENCES}

1. Brenton, J. D., Carey, L. A., Ahmed, A. A. \& Caldas, C. Molecular classification and molecular forecasting of breast cancer: ready for clinical application? J. Clin. Oncol. 23, 7350-7360 (2005).

2. Papadimitriou, M., Mountzios, G. \& Papadimitriou, C. A. The role of PARP inhibition in triple-negative breast cancer: Unraveling the wide spectrum of synthetic lethality. Cancer Treat. Rev. 67, 34-44 (2018).

3. Dent, R., Trudeau, M., Pritchard, K. I., Hanna, W. M., Kahn, H. K., Sawka, C. A. et al. Triple-negative breast cancer: clinical features and patterns of recurrence. Clin. Cancer 13, 4429-4434 (2007).

4. Bauer, K. R., Brown, M., Cress, R. D., Parise, C. A. \& Caggiano, V. Descriptive analysis of estrogen receptor (ER)-negative, progesterone receptor (PR)-negative, and HER2-negative invasive breast cancer, the so-called triple-negative phenotype: a population-based study from the California cancer Registry. Cancer 109, 1721-1728 (2007).

5. Bianchini, G., Balko, J. M., Mayer, I. A., Sanders, M. E. \& Gianni, L. Triple-negative breast cancer: challenges and opportunities of a heterogeneous disease. Nat. Rev. Clin. Oncol. 13, 674-690 (2016).

6. Lehmann, B. D., Bauer, J. A., Chen, X., Sanders, M. E., Chakravarthy, A. B., Shyr, Y. et al. Identification of human triple-negative breast cancer subtypes and preclinical models for selection of targeted therapies. J. Clin. Invest. 121, 2750-2767 (2011).

7. Burstein, M. D., Tsimelzon, A., Poage, G. M., Covington, K. R., Contreras, A., Fuqua, S. A. W. et al. Comprehensive genomic analysis identifies novel subtypes and targets of triple-negative breast cancer. Clin. Cancer Res. 21, 1688-1698 (2015).

8. Masuda, H., Baggerly, K. A., Wang, Y., Zhang, Y., Gonzalez-Angulo, A. M., MericBernstam, F. et al. Differential response to neoadjuvant chemotherapy among 7 triple-negative breast cancer molecular subtypes. Clin. Cancer Res. 19, 5533-5540 (2013).

9. Yarden, Y. \& Sliwkowski, M. X. Untangling the ErbB signalling network. Nat. Rev. Mol. Cell Biol. 2, 127-137 (2001).

10. Gonzalez-Conchas, G. A., Rodriguez-Romo, L., Hernandez-Barajas, D., GonzalezGuerrero, J. F., Rodriguez-Fernandez, I. A., Verdines-Perez, A. et al. Epidermal growth factor receptor overexpression and outcomes in early breast cancer: A systematic review and a meta-analysis. Cancer Treat. Rev. 62, 1-8 (2017).

11. Hoadley, K. A., Weigman, V. J., Fan, C., Sawyer, L. R., He, X., Troester, M. A. et al. EGFR associated expression profiles vary with breast tumor subtype. BMC Genomics 8, 258 (2007).

12. Costa, R., Shah, A. N., Santa-Maria, C. A., Cruz, M. R., Mahalingam, D., Carneiro, B. A. et al. Targeting epidermal growth factor receptor in triple negative breast cancer: new discoveries and practical insights for drug development. Cancer Treat. Rev. 53, 111-119 (2017)

13. Paik, S., Kim, C. \& Wolmark, N. HER2 status and benefit from adjuvant trastuzumab in breast cancer. N. Engl. J. Med. 358, 1409-1411 (2008).

14. Perez, E. A., Reinholz, M. M., Hillman, D. W., Tenner, K. S., Schroeder, M. J., Davidson, N. E. et al. HER2 and chromosome 17 effect on patient outcome in the N9831 adjuvant trastuzumab trial. J. Clin. Oncol. 28, 4307-4315 (2010).

15. Fehrenbacher, L., Jeong, J.-H., Rastogi, P., Geyer, C. E., Paik, S., Ganz, P. A. et al. OT1-02-07: NSABP B-47: A Randomized Phase III Trial of Adjuvant Therapy Comparing Chemotherapy Alone (Six Cycles of Docetaxel Plus Cyclophosphamide or Four Cycles of Doxorubicin Plus Cyclophosphamide Followed by Weekly Paclitaxel) to Chemotherapy Plus Trastuzumab in Women with NodePositive or High-Risk Node-Negative HER2-Low Invasive Breast Cancer. Cancer Res. 71(Suppl 24), OT1-02-OT1-007 (2011).

16. Fehrenbacher, L., Cecchini, R. S., Geyer, C. E., Rastogi, P., Costantino, J. P., Atkins, J. N. et al. Abstract GS1-02: NSABP B-47 (NRG oncology): Phase III randomized trial comparing adjuvant chemotherapy with adriamycin (A) and cyclophosphamide $(C) \rightarrow$ weekly paclitaxel (WP), or docetaxel (T) and C with or without a year of trastuzumab $(\mathrm{H})$ in women with node-positive or high-risk node-negative invasive breast cancer (IBC) expressing HER2 staining intensity of IHC $1+$ or $2+$ with negative FISH (HER2-Low IBC). Cancer Res. 78(Suppl 4), GS1-GS02 (2018).

17. Ilie, S. M., Desauw, C. \& Hebbar, M. HER2 based expression subpopulations in TNBC: pathological aspects and clinical significance. Ann. Oncol. 27(Suppl 6), vi15-vi42 (2016).

18. Bae, S. Y., La Choi, Y., Kim, S., Kim, M., Kim, J., Jung, S. P. et al. HER3 status by immunohistochemistry is correlated with poor prognosis in hormone receptornegative breast cancer patients. Breast Cancer Res. Treat. 139, 741-750 (2013).

19. Goldstein, N. S., Hewitt, S. M., Taylor, C. R., Yaziji, H. \& Hicks, D. G., Members of AdHoc Committee On Immunohistochemistry Standardization. Recommendations for improved standardization of immunohistochemistry. Appl. Immunohistochem. Mol. Morphol. 15, 124-133 (2007).

20. Perez, E. A., Press, M. F., Dueck, A. C., Jenkins, R. B., Kim, C., Chen, B. et al. Immunohistochemistry and fluorescence in situ hybridization assessment of HER2 in clinical trials of adjuvant therapy for breast cancer (NCCTG N9831, BCIRG 006, and BCIRG 005). Breast Cancer Res. Treat. 138, 99-108 (2013).

21. McCullough, A. E., Dell'orto, P., Reinholz, M. M., Gelber, R. D., Dueck, A. C., Russo, L. et al. Central pathology laboratory review of HER2 and ER in early breast cancer: an ALTTO trial [BIG 2-06/NCCTG N063D (Alliance)] ring study. Breast Cancer Res. Treat. 143, 485-492 (2014).

22. Mathis, G. HTRF(R) technology. J. Biomol. Screen 4, 309-314 (1999).

23. Ho-Pun-Cheung, A., Bazin, H., Gaborit, N., Larbouret, C., Garnero, P., Assenat, E. et al. Quantification of HER expression and dimerization in patients' tumor samples using time-resolved Förster resonance energy transfer. PloS ONE 7, e37065 (2012).

24. Lopez-Crapez, E., Ho-Pun-Cheung, A., Garnero, P. \& Bazin, H. Evaluation of the dimerization profiles of HER tyrosine kinases by time-resolved Förster resonance energy transfer (TR-FRET). Methods Mol. Biol. Clifton NJ 1233, 45-55 (2015).

25. Wolff, A. C., Hammond, M. E. H., Schwartz, J. N., Hagerty, K. L., Allred, D. C., Cote, R. J. et al. American Society of Clinical Oncology/College of American Pathologists guideline recommendations for human epidermal growth factor receptor 2 testing in breast cancer. Arch. Pathol. Lab. Med. 131, 18-43 (2007).

26. Albizu, L., Cottet, M., Kralikova, M., Stoev, S., Seyer, R., Brabet, I. et al. Timeresolved FRET between GPCR ligands reveals oligomers in native tissues. Nat. Chem. Biol. 6, 587-594 (2010).

27. McCarty, K. S., Szabo, E., Flowers, J. L., Cox, E. B., Leight, G. S., Miller, L. et al. Use of a monoclonal anti-estrogen receptor antibody in the immunohistochemical evaluation of human tumors. Cancer Res. 46(Suppl 8), 4244s-4248s (1986).

28. Silva, L. P., Lorenzi, P. L., Purwaha, P., Yong, V., Hawke, D. H. \& Weinstein, J. N. Measurement of DNA concentration as a normalization strategy for metabolomic data from adherent cell lines. Anal. Chem. 85, 9536-9542 (2013).

29. Lewin, B. Genes $V$ 5th edn. (Oxford University Press, Oxford, England, 1994).

30. DeSouza, L. V., Krakovska, O., Darfler, M. M., Krizman, D. B., Romaschin, A. D., Colgan, T. J. et al. mTRAQ-based quantification of potential endometrial carcinoma biomarkers from archived formalin-fixed paraffin-embedded tissues. Proteomics 10, 3108-3116 (2010).

31. Larson, J. S., Goodman, L. J., Tan, Y., Defazio-Eli, L., Paquet, A. C., Cook, J. W. et al. Analytical Validation of a Highly Quantitative, Sensitive, Accurate, and Reproducible Assay (HERmark) for the Measurement of HER2 Total Protein and HER2 
Homodimers in FFPE Breast Cancer Tumor Specimens. Pathol. Res. Int. 2010, 814176 (2010).

32. Rakha, E. A., El-Sayed, M. E., Green, A. R., Lee, A. H. S., Robertson, J. F. \& Ellis, I. O. Prognostic markers in triple-negative breast cancer. Cancer 109, 25-32 (2007).

33. Nakai, K., Hung, M.-C. \& Yamaguchi, H. A perspective on anti-EGFR therapies targeting triple-negative breast cancer. Am. J. Cancer Res. 6, 1609-1623 (2016).

34. Zhang, Q., Park, E., Kani, K. \& Landgraf, R. Functional isolation of activated and unilaterally phosphorylated heterodimers of ERBB2 and ERBB3 as scaffolds in ligand-dependent signaling. Proc. Natl Acad. Sci. USA 109, 13237-13242 (2012).

35. Capuani, F., Conte, A., Argenzio, E., Marchetti, L., Priami, C., Polo, S. et al. Quantitative analysis reveals how EGFR activation and downregulation are coupled in normal but not in cancer cells. Nat. Commun. 6, 7999 (2015).

36. Witton, C. J., Reeves, J. R., Going, J. J., Cooke, T. G. \& Bartlett, J. M. S. Expression of the HER1-4 family of receptor tyrosine kinases in breast cancer. J. Pathol. 200, 290-297 (2003).

37. Tanner, B., Hasenclever, D., Stern, K., Schormann, W., Bezler, M., Hermes, M. et al. ErbB-3 predicts survival in ovarian cancer. J. Clin. Oncol. 24, 4317-4323 (2006).

38. Ithimakin, S., Day, K. C., Malik, F., Zen, Q., Dawsey, S. J., Bersano-Begey, T. F. et al. HER2 drives luminal breast cancer stem cells in the absence of HER2 amplification: implications for efficacy of adjuvant trastuzumab. Cancer Res. 73, 1635-1646 (2013).

39. Phillips, T. M., McBride, W. H. \& Pajonk, F. The response of CD24(-/low)/CD44+ breast cancer-initiating cells to radiation. J. Natl Cancer Inst. 98, 1777-1785 (2006).

40. Shafee, N., Smith, C. R., Wei, S., Kim, Y., Mills, G. B., Hortobagyi, G. N. et al. Cancer stem cells contribute to cisplatin resistance in Brca1/p53-mediated mouse mammary tumors. Cancer Res. 68, 3243-3250 (2008).
41. Li, X., Lewis, M. T., Huang, J., Gutierrez, C., Osborne, C. K., Wu, M.-F. et al. Intrinsic resistance of tumorigenic breast cancer cells to chemotherapy. J. Natl Cancer Inst. 100, 672-679 (2008).

42. O'Hurley, G., Sjöstedt, E., Rahman, A., Li, B., Kampf, C., Pontén, F. et al. Garbage in, garbage out: a critical evaluation of strategies used for validation of immunohistochemical biomarkers. Mol. Oncol. 8, 783-798 (2014).

43. Steiner, C., Tille, J.-C., Lamerz, J., Kux van Geijtenbeek, S., McKee, T. A., Venturi, M. et al. Quantification of HER2 by targeted mass spectrometry in formalin-fixed paraffin-embedded (FFPE) breast cancer tissues. Mol. Cell Proteomics 14, 2786-2799 (2015).

44. Shi, Y., Huang, W., Tan, Y., Jin, X., Dua, R., Penuel, E. et al. A novel proximity assay for the detection of proteins and protein complexes: quantitation of HER1 and HER2 total protein expression and homodimerization in formalin-fixed, paraffinembedded cell lines and breast cancer tissue. Diagn. Mol. Pathol. 18, 11-21 (2009).

45. Desmedt, C., Sperinde, J., Piette, F., Huang, W., Jin, X., Tan, Y. et al. Quantitation of HER2 expression or HER2:HER2 dimers and differential survival in a cohort of metastatic breast cancer patients carefully selected for trastuzumab treatment primarily by FISH. Diagn. Mol. Pathol. 18, 22-29 (2009).

46. Nuciforo, P., Radosevic-Robin, N., Ng, T. \& Scaltriti, M. Quantification of HER family receptors in breast cancer. Breast Cancer Res. 17, 53 (2015).

47. Sperinde, J., Huang, W., Vehtari, A., Chenna, A., Kellokumpu-Lehtinen, P. L., Winslow, J. et al. p95HER2 methionine 611 carboxy-terminal fragment is predictive of trastuzumab adjuvant treatment benefit in the FinHer trial. Clin. Cancer Res. 24, 3046-3052 (2018).

48. Chumsri, S., Sperinde, J., Liu, H., Gligorov, J., Spano, J. P., Antoine, M. et al. High p95HER2/HER2 ratio associated with poor outcome in trastuzumab-treated HER2positive metastatic breast cancer NCCTG N0337 and NCCTG 98-32-52 (Alliance). Clin. Cancer Res. 24, 3053-3058 (2018). 